\title{
Nachwuchsförderung als wichtiger Baustein für wissenschaftliche Exzellenz
}

\author{
Kathrin Kottke \\ Passgenaue Programme und Vorhaben fördern die frühe Selbstständigkeit \\ junger Akademikerinnen und Akademiker.
}

Die Qualifizierung und Karriereentwicklung von talentierten Nachwuchswissenschaftlerinnen und -wissenschaftlern hat im Fachbereich Mathematik und Informatik der Westfälischen Wilhelms-Universität Münster (WWU) und im Exzellenzcluster Mathematik Münster einen hohen Stellenwert. Der Cluster, der ab Januar 2019 seine Arbeit aufnimmt, führt etablierte Maßnahmen des Fachbereichs weiter und baut darüber hinaus neue Vorhaben auf. Passgenaue Programme für verschiedene Karrierestufen stärken die frühe Eigenständigkeit und helfen dem wissenschaftlichen Nachwuchs, sein individuelles wissenschaftliches Profil zu schärfen. „Wir freuen uns sehr über den Erfolg des Exzellenzclusters und die Leistungen unserer Wissenschaftlerinnen und Wissenschaftler. Der Cluster schafft hervorragende Ausbildungsund Qualifizierungsbedingungen, von denen der wissenschaftliche Nachwuchs profitiert und seine akademische Laufbahn an exzellenter Forschung und Lehre ausrichten kann", betont Prof. Dr. Xiaoyi Jiang, Dekan des Fachbereichs Mathematik und Informatik.

Die breite mathematische Ausbildung an der WWU und regelmäßige interdisziplinäre Seminarangebote für Bachelor- und Masterstudierende sowie für Doktoranden ermöglichen, über den eigenen fachlichen Tellerrand zu schauen. Der Exzellenzcluster fördert darüber hinaus die internationale Mobilität von Master-Studierenden und Doktoranden, damit diese an renommierten Spitzenuniversitäten studieren können und neue Impulse für die eigene Arbeit erhalten. Zusätzlich lädt der Cluster hoch qualifizierte internationale Studierende und Doktoranden für ein Semester nach Münster ein. Die „Mathematics Münster Graduate School“ umfasst alle mathematischen Disziplinen an der WWU und integriert existierende Programme und Doktoranden am Fachbereich. „Die Graduate School bietet Promovierenden eine exzellente Qualifizierung und Betreuung durch international herausragende Wissenschaftlerinnen und Wissenschaftler. Zusätzlich haben Doktoranden die Chance, sich mit Kolleginnen und Kollegen anderer mathematischer Teildisziplinen auszutauschen und zu vernetzen", erklärt Prof. Dr. Wilhelm Winter, zukünftiger Leiter der Graduiertenschule. Auf Postdoktoranden-Ebene plant der Cluster, sowohl individuelle Stellen zu schaffen, als auch Nachwuchsgruppen einzurichten. Diese Vorhaben werden durch ein Konferenz- und ein Karriereentwicklungsprogramm für die interdisziplinäre Integration des Nachwuchses bei gleichzeitiger früher wissenschaftlicher Selbstständigkeit gestärkt.

Eine bedeutende Rolle in der Nachwuchsförderung der münsterschen Mathematiker kommt der Gleichstellung der Geschlechter zu. Mathematik gilt in Deutschland immer noch als ein eher „männliches“ Fach, obwohl bundesweit inzwischen 50 Prozent der Studierenden weiblich sind. Auf den höheren Qualifikationsebenen sind Frauen jedoch unterproportional häufig vertreten. In Bezug auf Gleichstellungsmaßnahmen sind bereits vielfältige chancenreiche Instrumente in Münster etabliert, die im Rahmen des Exzellenzclusters ausgebaut werden: Neben den Mentoring- und Netzwerkmaßnahmen werden weitere in Münster bewährte Programme für Akademikerinnen und für die Vereinbarkeit von Familie und Beruf ausgebaut. Unter anderem wird mit dem Ada-LovelaceStipendium bereits seit 2016 angestrebt, herausragende Mathematikerinnen für eine Promotion in Münster zu gewinnen. Jedes Jahr werden bis zu zwei Promotionsstellen finanziert. Das MATHRIX-JuniorprofessorinnenProgramm fördert seit 2017 zwei Mathematikerinnen durch Juniorprofessorinnenstellen mit einer attraktiven Ausstattung, um exzellente Forschung und Lehre zu ermöglichen.

Einblicke in die Forschung der beiden MATHRIX-Juniorprofessorinnen

Die beiden WWU-Mathematikerinnen Franziska Jahnke und Mira Schedensack beschreiben im Folgenden, was mathematische Forschung für sie bedeutet und welches Ziel ihre Forschung hat. Franziska Jahnke forscht auf dem Gebiet Modelltheorie von Körpern. Ihre Arbeit verbindet mathematische Logik mit Anwendungen in der Algebra. Ein großer Teil ihrer Arbeit beschäftigt sich mit der Shelah Vermutung über NIP Körper. Eine Grundlage hierfür ist das Studium der definierbaren Mengen in bewerteten Körpern. Mira Schedensack arbeitet auf dem Gebiet der Numerik partieller Differentialgleichungen, insbesondere zu Problemen aus der Kontinuumsmechanik. Im Vordergrund ihrer Forschung steht die Entwicklung neuer numerischer Verfahren und ihrer rigorosen a priori- und a posteriori-Fehleranalyse. Schwerpunkte liegen hierbei auf nicht-konformen und adaptiven Finite-ElementeMethoden. 


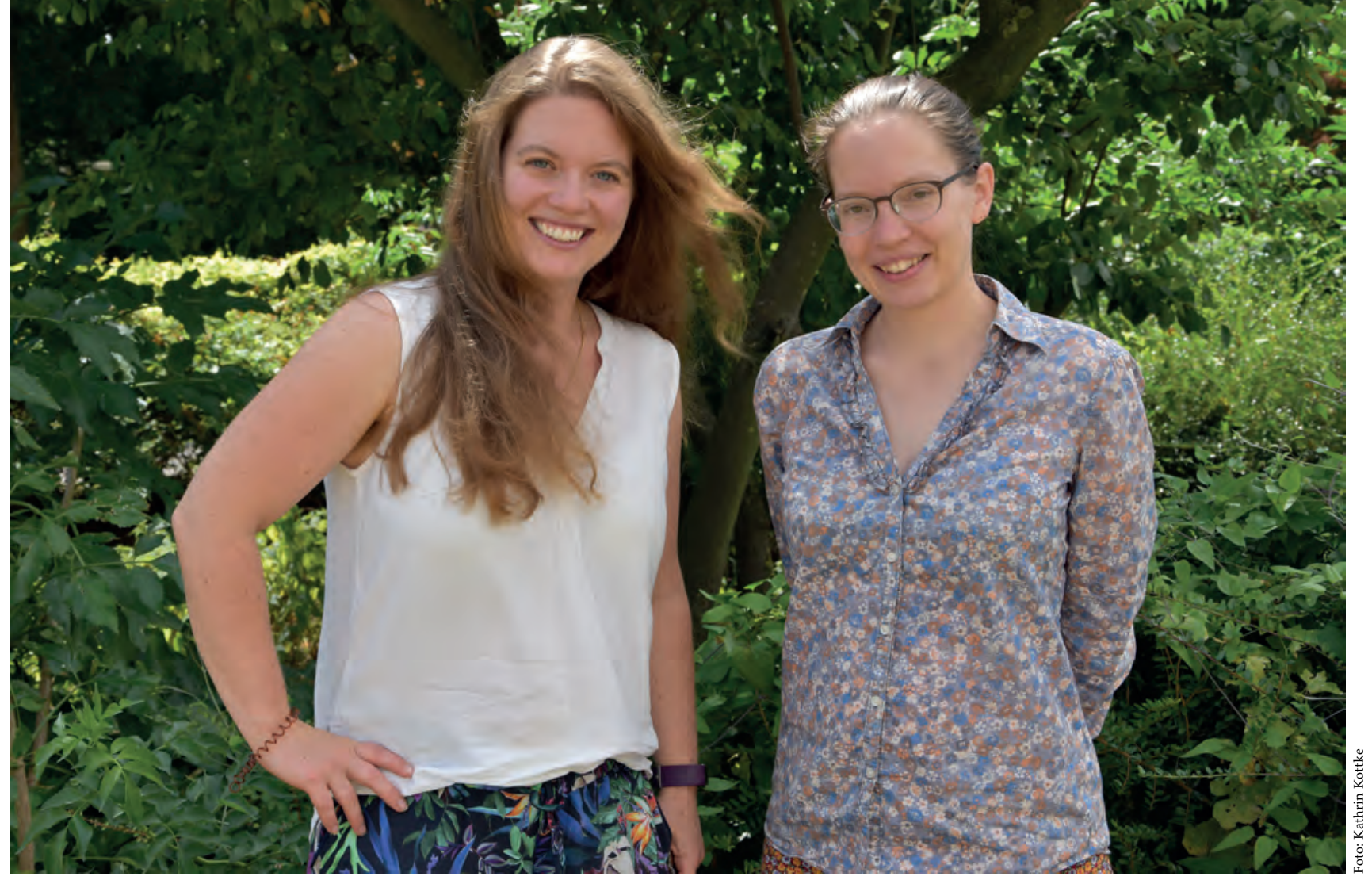

Die MATHRIX-Juniorprofessorinnen Franziska Jahnke und Mira Schedensack

Franziska Jahnke: „Die Augenblicke der Erkenntnis machen alle fruchtlos anmutenden Stunden wett"

Mathematische Forschung bedeutet für mich, gedankenversunken stundenlang ins Leere zu starren oder immer wieder neu auf ein Blatt Papier zu kritzeln. Natürlich lese ich auch Fachartikel, diskutiere mit meinen Ko-Autoren oder bringe meine Überlegungen zu Papier - aber das Kernstück meiner Arbeit sind doch die Stunden des Grübelns. Das ist häufig sehr frustrierend, immer wieder drehen sich meine Gedanken im Kreis ... Umso wertvoller ist der Moment, in dem eine Einsicht zu reifen beginnt. Banges Hoffen, ob sie den nun notwendigerweise folgenden Prüfungen standhält! Diese Augenblicke der Erkenntnis machen alle fruchtlos anmutenden Stunden zuvor wett und machen gleichzeitig Lust auf mehr.

Ich habe Zahlen, Knobeln und Rätsel schon als Kind gern gemocht. Zum Mathematikstudium hat mich das schöne Wetter nach Freiburg im Breisgau gelockt, wo ich mit der abstrakten Welt der reinen Mathematik in Berührung kam, die mich nicht mehr loslässt. Vor allem die Algebra, das Studium der Lösbarkeit von Gleichungen in verschiedenen Zahlsystemen, und die mathematische Logik, eine Grundlagendisziplin der Mathematik, haben es mir angetan. Nach dem Diplom habe ich mit einem Stipendium eines EU-Netzwerks an der Universität Oxford in Modelltheorie, einem Teilbereich der mathematischen Logik, promoviert. Das Stipendium ermöglichte es mir, zu vielen Konferenzen zu fahren und dabei Modell- theoretiker aus der ganzen Welt sowie ihre Forschungsgebiete kennenzulernen. Doch das Reisen kann das stille Kämmerlein nur ergänzen und nicht ersetzen! Nach der Promotion bin ich an die WWU Münster gekommen, wo ich erst als Assistentin und nun als Juniorprofessorin forsche und lehre. Seit zwei Jahren bin ich Mutter einer Tochter. Seitdem weiß ich: Auch auf einer Spielplatzbank lässt sich durchaus Mathematik betreiben!

In meiner Forschung studiere ich bestimmte Zahlensysteme aus der Algebra (bewertete Körper) mit Methoden der Modelltheorie. Insbesondere geht es dabei um Fragen wie „Wann beschreibt die Arithmetik des Körpers bereits die Bewertung, das heißt, welche Bewertungen sind definierbar?“ oder „Gibt es einen Zusammenhang zwischen den kombinatorischen Mustern, die in der Arithmetik des Körpers kodiert werden, und Eigenschaften der definierbaren Bewertungen auf dem Körper?". Zur zweiten Frage gibt es eine große und offene Vermutung von Shelah, einem berühmten israelischen Logiker. Hierbei geht es um NIP-Körper, das sind Körper in denen mittels der Arithmetik kein bipartiter Zufallsgraph beschrieben werden kann. Die Vermutung ist nun, dass jeder unendliche NIPKörper schon entweder algebraisch abgeschlossen (,wie die komplexen Zahlen'), reell abgeschlossen (,wie die reellen Zahlen') oder nicht-trivial henselsch bewertet (,wie die $p$-adischen Zahlen') ist. Ein Beweis oder die Widerlegung der Vermutung scheint noch unerreichbar, dennoch inspiriert und motiviert sie meine Forschung. In einem aktuellen Projekt mit meinen Ko-Autoren Yatir Halevi und 
Assaf Hasson (beide Universität Ben Gurion, Israel) zeigen wir, dass die Shelah-Vermutung eine andere bekannte Vermutung über NIP-Körper impliziert. In einem Projekt mit Sylvy Anscombe (University of Central Lancashire, UK) geben wir eine Klassifikation der henselschen NIP-Körper (modulo der Theorien der Restklassenkörper) an. In den letzten Jahren wurden erste kleine Teilerfolge beim Entschlüsseln der Shelah-Vermutung erzielt, unter anderem dank meiner Arbeit: Vor allem hat Will Johnson in seiner Promotion an der Universität Berkeley 2016 den eindimensionalen Fall gelöst, und dabei an zentraler Stelle ein Resultat von Jochen Koenigsmann (Universität Oxford) und mir zur Definierbarkeit $p$-henselscher Bewertungen benutzt. Dies nährt die Hoffnung, dass wir durch etliche weitere Stunden des Starrens einer Lösung in kleinen Schritten näherkommen.

Mira Schedensack: „Es gibt viele Anwendungsfelder, für die unsere Forschung relevant ist."

Wie berechnet ein Taschenrechner die Wurzel aus zwei? Schon bei dieser einfachen Aufgabe kommen numerische Verfahren zum Einsatz. Aus kaum einer Anwendung sind diese noch wegzudenken. Inwieweit man dem Ergebnis aber wirklich Vertrauen schenken darf, ist eine der Grundfragen der numerischen Mathematik, die für komplexere Probleme natürlich umso wichtiger ist. Mein Arbeitsgebiet ist die numerische Behandlung partieller Differentialgleichungen, wie sie zum Beispiel bei der Beschreibung von Phänomenen in den Natur- oder Ingenieurwissenschaften auftreten. Ein Beispiel für ein solches Phänomen ist die Wärmeverteilung in einem Raum: Die Temperatur in einem Ort hängt von der Temperatur in der ganzen Nachbarschaft ab. Durch diese Kopplung ist es im Allgemeinen nicht möglich, eine exakte Lösung zu berechnen. Deswegen werden Lösungen von partiellen Differentialgleichungen mit numerischen Verfahren approximiert, also angenähert. Seit meiner Promotionszeit an der Humboldt-Universität zu Berlin beschäftige ich mich mit der Entwicklung solcher Verfahren und ihrer Analyse. Es ist offensichtlich, dass solche Verfahren gewisse Kriterien erfüllen müssen. Beispielsweise sollte der Aufwand für die Umsetzung in einem Computerprogramm nicht zu groß sein. Da die Rechenkapazität begrenzt ist und mit vorhandenen Ressourcen - zum Beispiel dem
Arbeitsspeicher oder dem Stromverbrauch bei Hochleistungsrechnern - sparsam umgegangen werden sollte, liegt auch die Entwicklung effizienter numerischer Methoden im Fokus meiner Forschung.

Seit Oktober 2017 arbeite ich am Institut für Analysis und Numerik der WWU. Dort erforsche ich gemeinsam mit meinen Kollegen diese Methoden, um zum Beispiel folgende Fragen zu beantworten: Wenn eine Approximation gegeben ist, kann man dann den Fehler abschätzen, ohne dass man die exakte Lösung kennt? Kann man, ohne die exakte Lösung zu kennen, bestimmen, wie man eine Auflösung wählen muss, um die Lösung optimal zu approximieren? Das klingt für viele Personen zunächst abstrakt, aber es gibt tatsächlich viele Anwendungsfelder, für die unsere Forschung relevant ist - wenn man beispielsweise die Auslenkung einer Brücke unter einer Last simulieren möchte, um Schranken an kritischen Lasten zu berechnen.

Viele Algorithmen, die speziell bei komplizierten Problemen in der Industrie und anderen Anwendungen, zum Beispiel der Wettervorhersage, zum Einsatz kommen, können mit dem heutigen Wissensstand noch nicht gut analysiert werden. Daher ist eine gesunde Skepsis gegenüber Resultaten, die der Computer bereitstellt, auch immer wichtig. Oft wird in solchen komplizierten Problemen in Ermangelung mathematisch beweisbarer Fehlerabschätzungen heuristisch argumentiert. Dies kann dazu führen, dass sich die Numerik zu Beginn des Mathematikstudiums schwer einordnen lässt. Auch ich habe in meinem Studium die Numerik zunächst nur als Pflichtveranstaltung belegt. Dabei habe ich erfahren, dass mathematische Beweise im Mittelpunkt stehen. Die beweisbaren Resultate der Numerik beschränken sich häufig auf prototypische Gleichungen. Für diese können dafür aber Fehlerabschätzungen mathematisch hergeleitet werden. Mit diesen vor allem theoretischen Aspekten beschäftige ich mich auch in meiner jetzigen Forschung. Der mathematische Beweis, der im Mittelpunkt meiner Arbeitsweise steht, führt nicht nur auf rigorose Aussagen, sondern durch ihn wächst auch das Verständnis der betrachteten und noch zu entwickelnden Verfahren.

Ein Beispiel meiner Forschung ist eine aktuelle Kooperation mit der Ingenieurwissenschaft. Darin versuchen wir, gewisse Modelle aus der Elastizitätstheorie mit mathematisch fundierten Algorithmen anzugehen, wobei wir die mathematischen Grundlagen für die Simulation dieser Probleme erarbeiten.

JProf. Dr. Franziska Jahnke

Westfälische Wilhelms-Universität Münster,

Institut für Mathematische Logik und Grundlagenforschung,

Einsteinstraße 62, 48149 Münster

franziska.jahnke@uni-muenster.de

JProf. Dr. Mira Schedensack

Westfälische Wilhelms-Universität Münster,

Institut für Analysis und Numerik,

Orleansring 10, 48149 Münster

mira.schedensack@uni-muenster.de 